\title{
Programmable VCSEL-based photonic system architecture for future agile Tb/s metro networks
}

\author{
M. Svaluto Moreolo, ${ }^{1, *}$ (D) J. M. Fabrega, ${ }^{1}$ (D) L. NAdal, ${ }^{1}$ (i) R. Martínez ${ }^{1}$ \\ R. Casellas, ${ }^{1}$ (i) J. Vílchez, ${ }^{1}$ (i) R. Muñoz, ${ }^{1}$ (i) R. Vilalta, ${ }^{1}$ (i) A. Gatto, ${ }^{2}$ (i) P. Parolari, ${ }^{2}$ (i) \\ P. Boffi, ${ }^{2}$ C. Neumeyr ${ }_{y}{ }^{3}$ D. Larrabeiti, ${ }^{4}$ (D) G. Otero, ${ }^{4}$ and J. P. Fernández-Palacios ${ }^{5}$ \\ ${ }^{1}$ Centre Tecnològic de Telecomunicacions de Catalunya (CTTC/CERCA), Av. C. F. Gauss 7, 08860 Castelldefels (Barcelona), Spain \\ ${ }^{2}$ Politecnico di Milano, Via Ponzio 34/5, 20133 Milan, Italy \\ ${ }^{3}$ Vertilas GmbH, Daimlerstr. 11d, 85748 Garching, Germany \\ ${ }^{4}$ Universidad Carlos III de Madrid, Av. Universidad 30, 28911 Leganés, Spain \\ ${ }^{5}$ Telefonica R\&D, Edificio Sur 3, Planta 3, Distrito C 28050 Madrid, Spain \\ ${ }^{*}$ Corresponding author: michela.svaluto@cttc.es
}

\begin{abstract}
To deal with the challenging requirements of metropolitan area networks (MANs), it is essential to design costeffective systems that can support high capacity and dynamic adaptation, as well as a synergy of programmability and efficient photonic technologies. This becomes crucial for very large MANs that support 5G, where multihop connections will need to be dynamically established at target capacities beyond $\mathrm{Tb} / \mathrm{s}$. Programmability, automation, and modularity of network elements are key desired features. In this work, a modular photonic system, programmable via a software-defined networking platform, designed for dynamic 5G-supportive MANs, is described and analyzed. We consider modular sliceable bandwidth/bit rate variable transceivers (S-BVTs) based on vertical-cavity surface-emitting laser (VCSEL) technology and dense photonic integration. The proposed system and its programmability are experimentally assessed using a VCSEL with $10 \mathrm{GHz}$ bandwidth. The experiments are performed over connections as long as six-hop and $160 \mathrm{~km}$, from low-level aggregation nodes to metro-core nodes, thereby enabling IP off-loading. Furthermore, a numerical model is derived to estimate the performance when adopting higher bandwidth VCSELs $(\geq 18 \mathrm{GHz})$ and integrated coherent receivers, as targeted in the proposed system. The analysis is performed for both $50 \mathrm{GHz}$ and $25 \mathrm{GHz}$ granularities. In the former case, $50 \mathrm{~Gb} / \mathrm{s}$ capacity per flow can be supported over the targeted connections, for optical signal-to-noise ratio values above $26 \mathrm{~dB}$. When the granularity is $25 \mathrm{GHz}$, the filter narrowing effect severely impacts the performance. Nevertheless, $1.2 \mathrm{~Tb} / \mathrm{s}$ capacity (scalable to higher values if spectral/spatial dimensions are exploited) can be achieved when configuring the S-BVT to enable 40 VCSEL flows. This confirms that the system is promising to support $\mathrm{Tb} / \mathrm{s}$ connections in future agile MANs. (๑) 2020 Optical Society of America
\end{abstract}

\section{INTRODUCTION}

The metropolitan area network (MAN) is becoming one of the most challenging network segments to be addressed, due to stringent requirements in terms of traffic volume, traffic variance, and cost and power efficiency. With respect to volume, it is forecast that $33 \%$ of service provider network capacity will be in the metro segment by 2022 [1]. Regarding variance, traffic peaks move geographically during the day throughout the metro area due to commuting, business, and leisure activities. Finally, related to cost and power, it is of paramount importance to take into account the low capital and operational expenditures (CAPEX and OPEX) targeted by operators for the MAN segment, while maintaining a high quality of service (QoS) and bandwidth provisioning flexibility. Thus, in order to design a cost-effective ultrabroadband transport layer featuring dynamic capacity adaptation, novel architectures must be envisioned, seeking a synergy of programmability and efficient photonic technologies [2]. This will enable the MAN to deal with the challenges posed by, for example, the ever-increasing fiber-to-the-home (FTTH) rates, the support of $5 \mathrm{G}$ new radio backhaul and fronthaul traffic, and the scalable provisioning of $\mathrm{Gb} / \mathrm{s}$ mobile service at events with large crowds. Finally, it is also worth considering that content delivery networks (CDNs) and corporate virtual private networks (VPNs) will continue to generate large traffic flows traversing the MAN in the upcoming years. In fact, it is forecast that CDNs will represent $72 \%$ of Internet traffic by 2022 [1]. 
All of these requirements and derived constraints become crucial when considering very large 5G-supportive MANs, including a huge number of nodes at different aggregation levels. In fact, the target capacity to be dynamically supported can be on the order of $\mathrm{Tb} / \mathrm{s}$ and beyond, with very high variance. To face these MAN challenges, we propose a system architecture that adopts suitable photonic technologies combined with programmable and adaptive transport solutions. This includes scalable and modular multiflow multiformat/rate/reach transceivers intelligently driven from a software-defined networking (SDN) control platform. Among alternative options for reducing cost, power consumption and footprint, vertical cavity surface emitting laser (VCSEL) sources have emerged as a promising photonic technology for the MAN segment [2]. This is due to the cost-effectiveness and ease of mass producing and testing these devices, the availability of VCSELs operating at long wavelengths, as well as the efforts devoted to the optimization and implementation of designs with very high bandwidth ( $\geq 18 \mathrm{GHz}$ ) [3]. Furthermore, dense photonic integration and the ever-increasing maturity of photonic integrated circuit (PIC) technologies enable the design and implementation of very compact and efficient network elements in terms of cost, power consumption, and footprint [2,4].

Among the different network elements, the transceiver represents a key enabler for high capacity and flexible, agile, and dynamic MANs. In particular, a programmable sliceable bandwidth/bit rate variable transceiver (S-BVT) enables a set of functionalities and abilities suitable for MANs, such as multiflow generation, "slice-ability," adaptability to traffic and reach, inverse multiplexing operation, and spectrum fragmentation mitigation, among others $[2,5,6]$. Indeed, the requirements and constraints of MANs should be carefully taken into account for a proper S-BVT design and implementation. A promising solution should fully exploit the available resources and the spectral/spatial dimensions. To this extent, we propose adopting cost-effective VCSEL-based S-BVTs integrated on a PIC, supporting up to $2 \mathrm{~Tb} / \mathrm{s}$. The S-BVT architecture is scalable to higher capacity by means of a modular approach and exploiting multiple dimensions $[7,8]$.

The modularity of the proposed design enables the upgrade and composition of the architecture, following a grow-asneeded approach and/or pay-as-you-grow scheme. Also, a license-based module activation scheme can be considered for a dense photonic integrated transceiver solution composed of multiple modules. This also allows the solution to be easily scaled to multiple dimensions. Particularly, additional fibers or multicore fibers (MCFs) can be used to target very high capacity support beyond $100 \mathrm{~Tb} / \mathrm{s}$. An additional benefit of the modular approach is the facilitation of disaggregation and multi-vendor interoperability, while lowering CAPEX and OPEX $[9,10]$. In this context, the programmability and automation of the network elements are key, as well as the agility of the S-BVT in terms of transmitter (Tx) and receiver $(\mathrm{Rx})$ configuration $[9,10]$. Thus, the use of an SDN platform is proposed to enable flexible, efficient, and dynamic exploitation of the optical layer through smart/agile S-BVT configuration, traffic aggregation, and statistical multiplexing. The most challenging goal of the envisioned system is the support of very high capacity (beyond $1 \mathrm{~Tb} / \mathrm{s}$ ) all-optical circuits across the multiple hierarchical levels (HLs) of the MAN to meet the traffic transport challenges described above. In the most relevant settings, these paths can have six hops on average, and in the worst case, can reach about $150 \mathrm{~km}$, as further explained in the following section.

In [11], we have analyzed the case of multiple-HL connectivity in a 5G-supportive MAN based on real topologies [12], considering six aggregation nodes with different functionalities and a 160-km-length path. The performance, adopting VCSEL-based S-BVTs, was assessed and showed that the coherent receiver (CO-Rx) module option outperforms direct detection (DD), enabling promising results for multihop MAN connections.

This paper is an extension of the work reported in [11], aimed at further explaining and demonstrating the suitability of the proposed system architecture as a promising solution for a MAN evolutionary scenario. The reference dynamic 5Gsupportive MAN and related parameters of interest, as well as use-case and worst-case analysis, not included in [11], are here reported. The system architecture is described in detail. Special focus is devoted to the adopted technologies and their effectiveness (in terms of cost, footprint, and power consumption) for an actual implementation, as envisioned in the framework of the H2020 PASSION project [13]. An entire section has been included towards further demonstrating that the proposed system can cope with upcoming MAN needs. This section includes a completely new analysis, using the experimental results of [11] (obtained with a $10 \mathrm{GHz}$ VCSEL) as a starting point. A numerical model is derived to evaluate the expected performance if higher bandwidth VCSELs and integrated CO-Rx modules are adopted, as envisioned for the proposed photonic system, to target $\mathrm{Tb} / \mathrm{s}$ MAN connections. In addition, a comparison with a theoretical model, developed for the proposed system, is provided. A broader analysis (including different node granularities) and discussion of the obtained results are also presented. Furthermore, additional details with respect to [11] are provided regarding the programmability and control aspects, including the adopted technology limitations, features, and functionalities.

The paper is structured as follows. Section 2 describes the dynamic 5G-supportive MAN that is considered for dimensioning and designing the system architecture. It also provides details on the use cases that are considered, which motivate this work. In particular, the characterization of the path of interest and the worst-case parameters are derived. Section 3 describes the photonic system architecture, designed to target cost-effective ultrabroadband transport, featuring dynamic capacity adaptation for the $5 \mathrm{G}$-supportive MAN. Special focus is devoted to the VCSEL-based S-BVT and the proposed technological solutions. Section 4 describes the experimental setup that was implemented adopting a single-flow S-BVT based on $10 \mathrm{GHz}$ VCSEL technology and using either DD or CO-Rx options. The experimental results obtained for assessing the connectivity performance of the system are also reported for up to six-hop and $160 \mathrm{~km}$ paths, including $25 \mathrm{~km} \mathrm{19-core} \mathrm{MCF.}$ Section 5 is devoted to theoretically and numerically modeling the proposed system. A comparison with the obtained experimental results is provided. Then, based on the derived numerical model, the expected performance is estimated for 

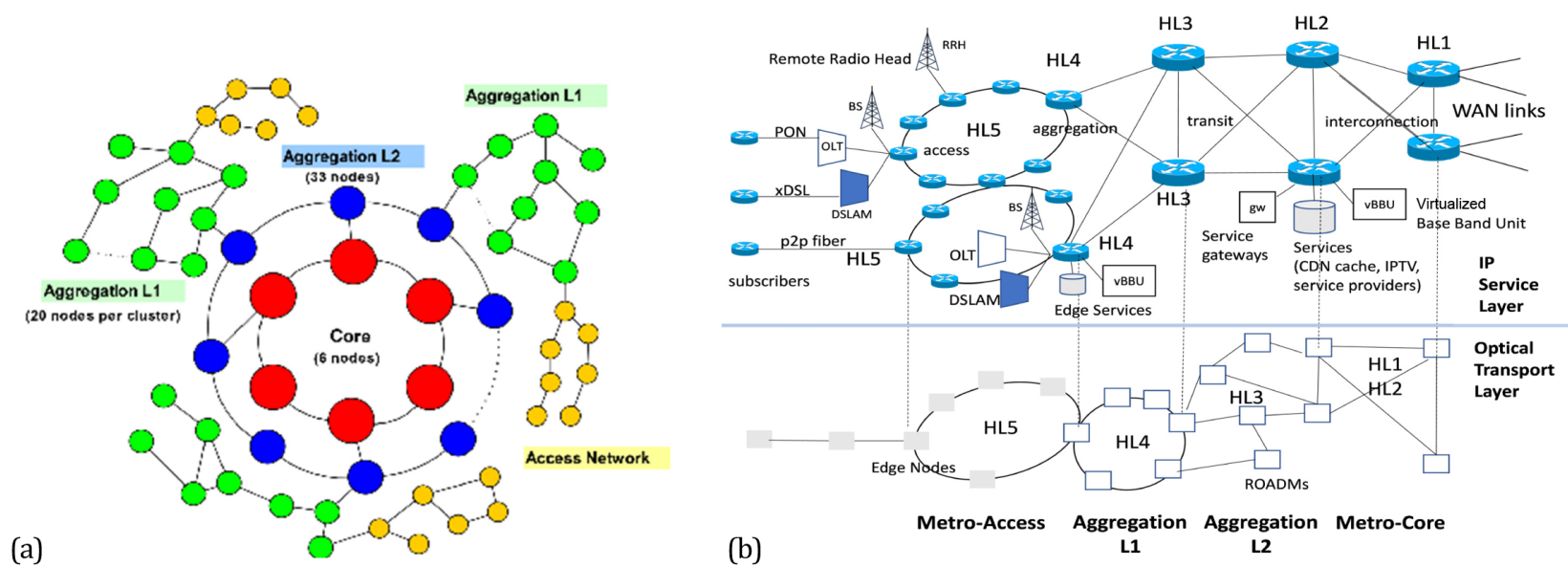

Fig. 1. (a) Structure of the reference network based on a very large Telefonica MAN, including access, aggregation level L1, aggregation level L2, and the core level. (b) Schematic view of the network hierarchical structure; the optical transport layer and IP layer are indicated.

the scenario where improved photonic and optoelectronic components are adopted. Specifically, the impact on the supported capacity is analyzed when adopting larger bandwidth VCSEL sources and an integrated CO-Rx. Different granularities of the switching node filters are considered. Section 6 deals with the system programmability and control aspects. Finally, in Section 7, conclusions are drawn.

\section{DYNAMIC 5G-SUPPORTIVE MAN}

A very large MAN, corresponding to Telefonica's reference network architecture, is considered. It provides connectivity service to a vast metropolitan area with 25 million inhabitants (specifically, it refers to Mexico City). This reference MAN is composed of a very large number of nodes (over 2800) and links. A simplified structure is represented in Fig. 1(a), and a schematic view of the hierarchical structure is displayed in Fig. 1(b). At the top level of the hierarchy, HL1 nodes provide WAN connectivity, whilst HL2 nodes host the MAN services, such as CDN caching (e.g., Movistar IPTV, Netflix, Amazon video). At the bottom of the hierarchy, HL5 access nodes are small points of presence and $5 \mathrm{G}$ base stations (i.e., gNBs). The purpose of the physical layer topology is the support of IP layer connectivity, as depicted in Fig. 1. The reference topology is based on current real subtopologies, with an average nodal degree of two in the access (ring-like) and five (mesh) in the core. However, to exploit the advantages of reconfigurable optical add/drop multiplexer (ROADM) nodes, nodal degrees greater than two are expected to enrich the connectivity at HL4 and HL3. This will lead to the eventual rollout of a mesh network topology, enabling multiple disjoint paths that can be deployed for interconnecting different HLs.

An estimate of the traffic for the year 2030 is made for this reference network by using (i) a figure of $20 \mathrm{~Gb} / \mathrm{s}$ of $5 \mathrm{G}$ backhaul traffic per HL5 (2432 access nodes) and HL4 node (380 central offices), (ii) FTTH to 5M Internet subscribers with 10:1 oversubscription ratios for $1 \mathrm{~Gb} / \mathrm{s}$ bidirectional service, and (iii) enterprise connectivity services at $10 \mathrm{~Gb} / \mathrm{s}$ (7000 medium-sized enterprises requiring Internet and VPN connectivity packages) without oversubscription. This results in a $1 \mathrm{~Tb} / \mathrm{s}$ peak data rate at HL4 (aggregation layer 1). This worst-case peak data rate results in over $50 \mathrm{~Tb} / \mathrm{s}$ of traffic per HL2 or HL1 core node (four and two nodes, respectively, in our sample network), considering that $90 \%$ of MAN traffic is hierarchical (i.e., Internet access/CDN/IPTV/WAN VPN, hosted by HL1 and HL2 nodes). Thereby, assuming that an operator's network could be designed to work in a traffic load range of $10 \%-50 \%$, the average aggregated traffic per central office in 2030 is expected to range from $100 \mathrm{~Gb} / \mathrm{s}$ to $500 \mathrm{~Gb} / \mathrm{s}$ and, in the core nodes, from 5 to $25 \mathrm{~Tb} / \mathrm{s}$. If $5 \mathrm{G}$ new radio fronthaul traffic (rather than backhaul traffic) is transported throughout the MAN, then this analysis is conservative because new radio and massive MIMO may yield $\mathrm{Tb} / \mathrm{s}$ rates per HL5 [6]. Note that this scenario provides another application niche for S-BVTs in the access metro segment for interconnecting HL5 and HL4 nodes.

In this evolutionary MAN scenario, two all-optical interconnection approaches become relevant. On the one hand, a design choice is the all-optical interconnection of two adjacent aggregation levels. For instance, an HL4 is directly connected to its closest HL3, building the logical direct links HL4HL3, as depicted in Fig. 1(b). On the other hand, it would be extremely advantageous to support all-optical interconnection of any HL4 node with the core level (HL1/HL2), taking into account that most of the MAN traffic (approximately 90\%, according to Telefonica's internal statistics) is hierarchical; i.e., it is steered towards the core network segment. By doing so, IP off-loading of HL3 traffic onto the optical layer can be attained. For the remaining $10 \%$ of intralevel traffic, the slicing capability of the S-BVT provides an excellent choice for interconnection. In fact, with a single transceiver, an HL4 router can be connected at high rates directly to the core routers (i.e., HL2/HL1), and eventually at lower speeds with the neighboring HL4 and/or HL3 nodes.

All-optical transport reduces the number of IP routers, leading to a notable cost reduction. Specifically, the cost of high-end packet switching would be avoided in HL3, resulting in a convenient option for the network operator. This is enabled by establishing multihop all-optical connections from 
Table 1. Primary and Secondary Path Characterization for HL4 Node Connections to HL2/1 and HL3 Nodes

\begin{tabular}{lcccccccc}
\hline \multirow{2}{*}{$\begin{array}{l}\text { HLnnected } \\
\text { HLs }\end{array}$} & \multicolumn{3}{c}{ \# Hops } & & \multicolumn{3}{c}{ Length (km) } \\
\cline { 2 - 4 } & Mean & Std. Dev. & Max & & Mean & Std. Dev. & Max \\
\hline $\begin{array}{l}\text { HL4-HL2/1 } \\
\text { (primary) }\end{array}$ & 4.1 & 1.4 & 8 & & 36.1 & 25.2 & 131.3 \\
$\begin{array}{l}\text { HL4-HL2/1 } \\
\text { (secondary) }\end{array}$ & 7 & 2.2 & 14 & 74.7 & 29.5 & 148.4 \\
$\begin{array}{l}\text { HL4- HL3 } \\
\text { (primary) }\end{array}$ & 2.6 & 0.9 & 5 & & 18.3 & 11.7 & 64.5 \\
$\begin{array}{l}\text { HL4- HL3 } \\
\text { (secondary) }\end{array}$ & 6 & 1.8 & 9 & & 45.4 & 18.9 & 78.7 \\
\hline
\end{tabular}

HL4 to HL2/1. Of course, this use case poses the most challenging network setting in terms of transmission and switching requirements. In fact, the adopted technologies should be able to support ultrabroadband traffic over longer paths, adding and dropping traffic at many intermediate nodes. Thus, identifying a representative target worst path (to be covered in the reference topology) is of paramount relevance to assess the suitability of the proposed technologies and solutions.

In [12], a procedure for the path characterization, in terms of fiber length and number of hops, has been addressed considering the semisynthetic large MAN described above. Table 1 provides some of the findings. All-optical primary and secondary paths for HL4-HL2/1 and HL4-HL3 connections are considered. That is, any HL4 node is connected to the first or second closest HL2/1 and HL3 node, respectively, with the stringent requirement that the secondary paths must be physically disjoint from primary paths, for protection purposes. Both (primary and secondary) paths are obtained, minimizing the number of hops (hop-shortest disjoint path), to reduce the accumulated impairments while traversing the nodes (e.g., filter narrowing effect, insertion loss). This can become critical depending on the specific node architecture $[8,14]$.

It is important to observe that, from the performed study and assuming a normal distribution of path lengths, $68 \%$ of primary and $50 \%$ of secondary HL4-HL2 paths have fewer than six to seven hops, while the maximum length of any secondary path is about $150 \mathrm{~km}$ (see Table 1). In the case where each HL4 node is connected to the second-closest HL3 node, over the hop-shortest disjoint path, the average hop number is six with a length below $50 \mathrm{~km}$. These statistics motivate the analysis of six-hop connections with maximum path lengths of $150 \mathrm{~km}$. To address this scenario with cost-effective broadband technologies, a suitable photonic system architecture has been designed, as detailed in the following sections, and its effectiveness in supporting the targeted performance is discussed.

\section{SDN-ENABLED MODULAR PHOTONIC SYSTEM ARCHITECTURE}

In this section, the photonic system architecture designed for addressing the dynamic 5G-supportive MAN and related challenges is described. It is based on the EU-H2020 PASSION project approach [13]. Particularly, in this section, we describe the technologies targeted to be adopted and implemented in this framework.

The lower level metro-aggregation nodes (at HL4) are equipped with simple and cost-effective transceiver and switching technology, exploiting only the spectral dimension. Higher-level metro-core nodes (at HL2/1) adopt full-featured high-capacity transceivers and photonic switching (PS) nodes, supporting multiple functionalities and granularities, while handling both the spectral and spatial dimensions. The photonic system elements are composed of multiple fundamental blocks. They feature a modular approach to enable a pay-as-you-grow model and ease disaggregation [2,9].

As more accurately detailed in the following subsections, the transceivers are programmable S-BVTs based on direct multicarrier modulation (MCM) of long-wavelength, short-cavity (SC) VCSELs. This enables the support of up to $50 \mathrm{~Gb} / \mathrm{s}$ per flow at the Tx side. Then, according to the traffic required to be supported over an established lightpath, the corresponding VCSEL source(s), operating at the appropriate wavelength(s), are enabled/disabled by the SDN controller for a dynamic path/capacity adaptation.

At the Rx side, coherent detection is considered, since it supports improved performance in terms of achievable reach and robustness against transmission impairments with respect to DD, which in turn ensures a more cost-effective design. S-BVTs and nodes are programmable by an SDN controller via specific SDN agents, as shown in Fig. 2 and detailed in Section 6. The S-BVT Tx/Rx are appropriately configured, as well as the nodes, over the established path, according to their specific functionalities and programmable parameters.

As specified in [8], HL4 nodes are low-cost switches that enable simple add/drop functionality. They consist of $100 \mathrm{GHz}$ or $50 \mathrm{GHz}$ multiplexers/demultiplexers, based on arrayed waveguide gratings (AWGs), splitters, and semiconductor optical amplifiers (SOAs), acting as wavelength blockers, to aggregate/drop traffic in the spectral domain from/to lower level (HL5) nodes. HL2/HL1 nodes, similar to the transit HL3 nodes, have a more complex architecture. They include more functionalities enabled by multiple fundamental blocks [8], which are (i) the add switch, (ii) the aggregate/disaggregate switch, (iii) the multicast switch (MCS), and (iv) the PS module. The add switch provides aggregation of the flows from the S-BVTs, or from the aggregate/disaggregate switch, to the add ports of the PS module. The aggregate/disaggregate switch allows dropping traffic towards the MCS, which in turn drops the flows towards the S-BVT Rx modules. The aggregate/disaggregate switch also allows the addition of traffic flows in the node by means of the add switch. The PS module performs space switching of the

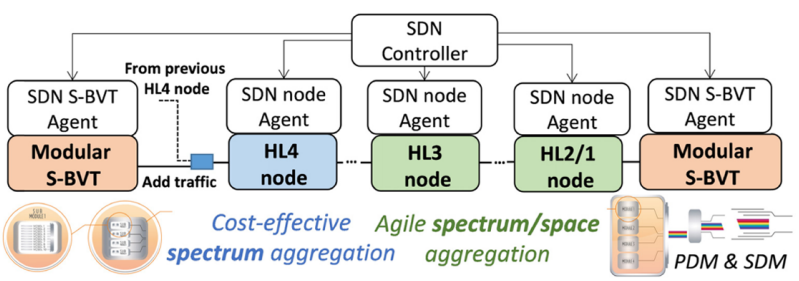

Fig. 2. Schematic of the programmable photonic system architecture. 
traffic from any input port to any output port, supporting both express and dropped paths. This allows an efficient usage of the network spectral and spatial resources. In fact, wavelengthselective switches (WSSs) are adopted to handle spectrum aggregation with a granularity of $25 \mathrm{GHz}$, and a polymer-based PS module to handle the spatial dimension $[8,15]$. The WSS can be implemented using either an InP monolithic approach or a hybrid approach adopting InP for active elements (SOAs) and SiPh for passives [16]. Modular upgradability is achieved repeating the same fundamental blocks; the interconnection with multicore fibers is performed via fan-in and fan-out structures.

\section{A. Modular VCSEL-Based Photonic Multi-Tb/s S-BVT}

In this section, an inner view of the S-BVT architecture is provided. The basic element is a directly modulated InP VCSEL with operating wavelength within the C-band, thanks to the buried tunnel junction (BTJ). An optimized structure [as depicted in Fig. 3(a)] allows single-mode operation to be obtained, with a side-mode suppression ratio higher than $35 \mathrm{~dB}$ (typical value of about $40 \mathrm{~dB}$ ) and a high modulation bandwidth beyond $18 \mathrm{GHz}$. This is achieved thanks to a very short effective cavity length $(2.5 \mu \mathrm{m})$, ensuring a reduced photon lifetime within it, distributed Bragg reflectors (DBRs) featuring short penetration depth, and an optimized active region, based on highly compressively strained AlGaInAs/InGaAs quantum wells [7]. The parasitic effects are reduced by the benzocyclobuthene (BCB) spacer layers. The device is characterized by a typical aperture of $5 \mu \mathrm{m}$, low power consumption $(<30 \mathrm{~mW})$, bias current in the range of 5-10 $\mathrm{mA}$, and $7 \mathrm{dBm}$ of output power. The linewidth (about $5 \mathrm{MHz}$ ) enhancement factor is about 3 (measured chirp $\alpha$ value 3.7 and adiabatic constant $\kappa 1.52 \cdot 10^{13}$ ) [17]. In addition to the low power consumption, the small footprint of this optimized VCSEL device enables dense photonic integration. Particularly, efficient coupling (loss $<0.5 \mathrm{~dB}$ ) to a silicon (Si) waveguide can be obtained with $45^{\circ}$ total internal reflection (TIR) mirrors $[4,18]$.

The fundamental integrated S-BVT module (MOD) consists of 40 VCSELs. It is composed of four submodules

(a)

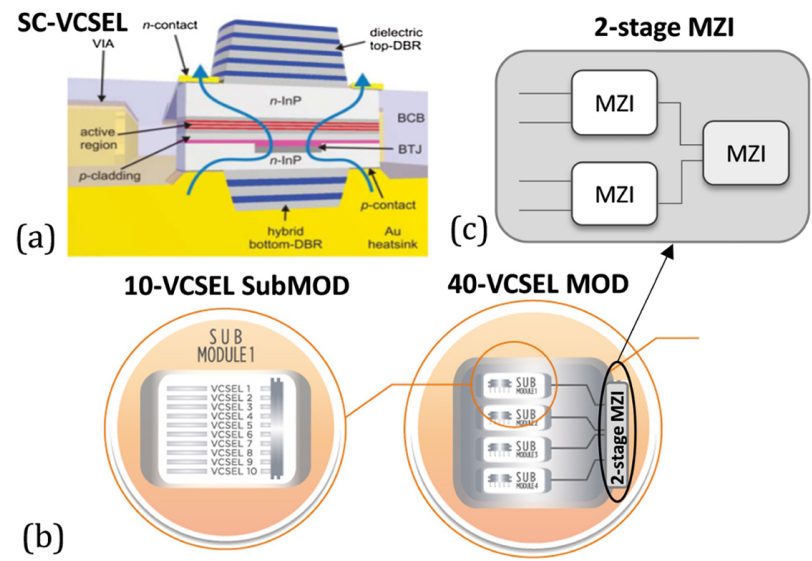

Fig. 3. (a) SC-VCSEL structure optimized for high modulation bandwidth, (b) fundamental S-BVT MOD composed of four subMODs with 10 VCSELs each, (c) two-stage MZI.
(subMODs) with 10 VCSELs each, integrated on a silicon-oninsulator (SOI) chip. A PIC platform, based on 3- $\mu$ m-thick SOI waveguides, is exploited to ensure low loss, high optical power throughput, and broadband operation. Furthermore, dense integration and massive production are facilitated [4]. The chip of $4 \mathrm{~cm}^{2}$ includes four AWGs, with $400 \mathrm{GHz}$ free spectral range (FSR), multiplexing the VCSEL channels of the four subMODs, and a two-stage Mach-Zehnder interferometer (MZI) interleaving them, providing an aggregated flow, coupled to the output fiber, of $100 \mathrm{GHz}$ spaced channels, as detailed in [18]. They cover the C-band at operating frequencies in the range $191.900-195.875 \mathrm{THz}$, corresponding to the ITU-T grid channels $\mathrm{CH} 19-\mathrm{CH} 58$. As described in the next subsection, by adopting a suitable digital signal processing (DSP), each VCSEL flow can achieve capacities up to $50 \mathrm{~Gb} / \mathrm{s}$. Thus, the 40-VCSEL SOI chip (MOD) is able to support up to $2 \mathrm{~Tb} / \mathrm{s}$ with power consumption lower than $5 \mathrm{pJ} / \mathrm{bit}$.

The spectral resources can be fully exploited, closely packing the VCSEL flows with $25 \mathrm{GHz}$ spacing, filtering out one sideband of the intensity modulated channels. This is obtained by multiplexing four fundamental modules with VCSEL operating wavelengths suitably tuned to have $25 \mathrm{GHz}$ spacing. In fact, the VCSELs in a module can be fine-tuned in a range of $75 \mathrm{GHz}$ through the bias current and stabilized by a temperature cooler $[16,18]$. The optical flows originating from the four different 40-VCSEL modules are then aggregated in the spectral domain [18]. Identifying each S-BVT VCSEL with the tuple formed by VCSEL-id/subMOD-id/MOD-id (with the corresponding id number, as in Fig. 4), a VCSEL with operating wavelength $191.900 \mathrm{THz}$ is the first VCSEL (VCSEL-1) of the first submodule (subMOD-1) of the first module (MOD-1). The adjacent (25 GHz-spaced) one is the first VCSEL of the first subMOD of the second MOD (VCSEL-1/subMOD-1/MOD-2), etc. It is important to note that the fundamental element integrated on a single PIC is the 40-VCSEL module. In order to prevent uncontrolled thermally induced wavelength shifts, accurate active and passive thermal paths allow constant PIC temperature operations and large cooling temperature excursion of VCSEL drivers [18]. To minimize their power consumption, the PIC and VCSELs have been designed to operate at a temperature that is a reasonable trade-off to balance the optical output power and overall power of the externally mounted thermoelectric cooler (TEC) [18].

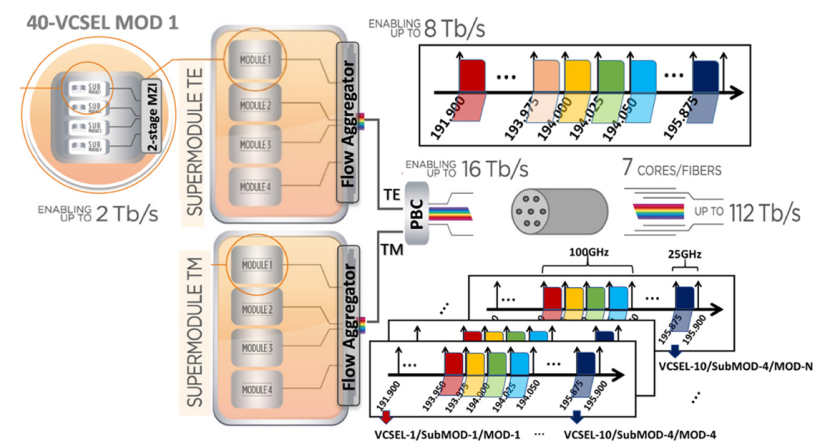

Fig. 4. Supermodule composition and scaling to polarization and spatial dimensions. In the top and bottom insets (on the right): wavelength assignment of VCSELs (with the corresponding identifier) to the different modules. 
The obtained fully equipped S-BVT Tx, also referred to as a supermodule, covers the $\mathrm{C}$-band range with $25-\mathrm{GHz}$ spaced channels (at $50 \mathrm{~Gb} / \mathrm{s}$ each) and enables supporting up to $8 \mathrm{~Tb} / \mathrm{s}$. As shown in Fig. 4, this capacity can be doubled, exploiting polarization division multiplexing (PDM). A supermodule transverse electric (TE) and a supermodule transverse magnetic (TM) adopting a total of eight fundamental modules are multiplexed with a polarization beam combiner (PBC) providing a total capacity of $16 \mathrm{~Gb} / \mathrm{s}$. To improve the capacity by a factor $M$, the space dimension can be exploited (space division multiplexing, SDM) adopting a bundle of $M$ fibers or an MCF with $M$ cores. For example, by adopting a seven-core MCF, a capacity above $100 \mathrm{~Tb} / \mathrm{s}$ can be achieved. At the Rx side, photonic integrated CO-Rx modules are envisioned to improve the capacity/reach performance. Nevertheless, for a further cost/complexity reduction of the S-BVT design, DD also can be considered [11].

\section{B. Adaptive MCM DSP}

For maximizing the system capacity and flexibility while enabling fine granularity, MCM is adopted at the transceiver DSP. Either discrete multitone (DMT) or orthogonal frequency division multiplexing (OFDM) can be implemented by suitably selecting the DSP blocks, as indicated in Fig. 5 and according to the Tx and Rx optoelectronic front ends [2,19]. Single sideband (SSB) modulation is preferred to double sideband MCM, since it enables higher spectral efficiency and $25 \mathrm{GHz}$ granularity, fully exploiting the SC-VCSEL bandwidth $[7,17]$. An optimal (or suboptimal) bit-loading (BL) algorithm is applied at the mapper to adaptively assign the appropriate bit number to each subcarrier. Similarly, power loading (PL) also can be adaptively applied to each subcarrier. This strategy enables one to finely manipulate the spectrum with the granularity of tens of megahertz, depending on the number of adopted MCM subcarriers. For example, in the case of using 512 subcarriers occupying $16 \mathrm{GHz}$ bandwidth, the resulting granularity is about $30 \mathrm{MHz}$. Furthermore, the loading algorithm allows maximizing the performance at a fixed capacity (margin adaptive, MA) or maximizing the capacity at a target performance (rate adaptive, RA). The assignment is performed according to the channel state information (CSI) retrieved at the $\mathrm{Rx}$ side. The specific values of the signal-tonoise ratio (SNR) per subcarrier are obtained applying uniform loading (UL) with the same modulation (4QAM) format for all of the MCM subcarriers at the Tx.

Figure 5 details the MCM fundamental blocks. They include at the Tx serial-to-parallel conversion (S/P), mapping of the digital/electrical subcarriers, addition of training symbols (TSs), inverse fast Fourier transform (IFFT), addition of the cyclic prefix $(\mathrm{CP})$, and parallel-to-serial $(\mathrm{P} / \mathrm{S})$ conversion. The baseband signal is clipped and can be upconverted to an intermediate radio frequency (RF). Before the digital-to-analog converter (DAC), a pre-emphasis block can also be applied to obtain improved performance, taking into account the DAC transfer function profile. The same steps in reverse order are followed at the Rx, for retrieving the transmitted data from the analog-to-digital converter (ADC): the OFDM signal is downconverted to baseband (if applicable), and a sync and retiming

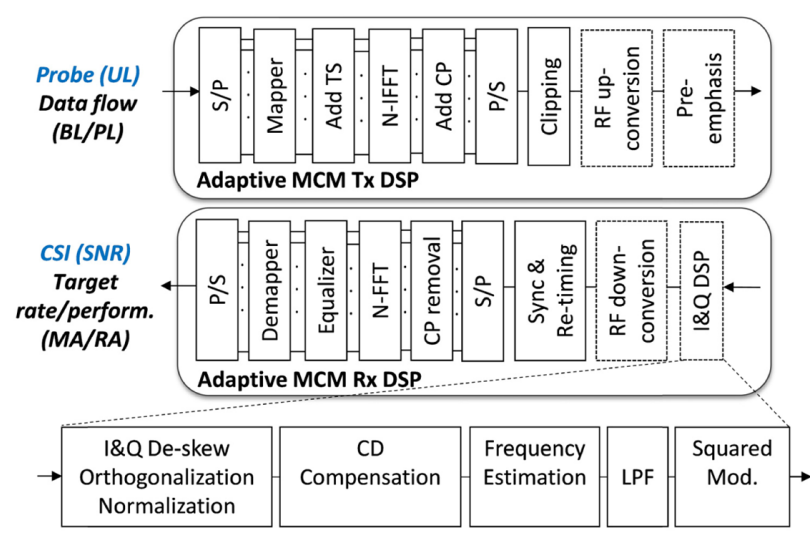

Fig. 5. Tx and Rx DSP based on adaptive MCM, in the bottom inset specific DSP for recovering $I$ and $Q$ components with a CO-Rx. The DSP blocks with the dashed line are optional, depending on the selected optoelectronic scheme.

(downsampling) procedure is applied to obtain a suitable baseband OFDM signal, then S/P is applied, CP removed, and the FFT performed; finally, the signal is equalized, demapped, and converted to serial. These steps (DSP blocks) are used for both $\mathrm{DD}$ and a CO-Rx.

In the case of adopting a CO-Rx, even if a simplified scheme is utilized, due to direct modulation (DM) at the $\mathrm{Tx}$, the $\mathrm{Rx}$ DSP includes additional modules for the appropriate processing of the in-phase and quadrature (I/Q) components of the MCM signal/flow, as shown in Fig. 5. In fact, the digitized I/Q components are first deskewed, orthogonalized, and normalized in order to optimally combine them, obtaining a proper complex representation of the received signal. This DSP is important for the performance of any CO-Rx. Of course, the use of an integrated $\mathrm{Rx}$ front end is advantageous, since delays in the different paths of the balanced detectors can be appropriately designed and manufactured. However, when using a $\mathrm{Rx}$ with discrete components, this DSP module is key for compensating for the differences between I/Q components and obtaining a proper complex representation of the received signal.

Afterwards, thanks to the adoption of coherent reception, the accumulated chromatic dispersion (CD) can be easily compensated, by employing a suitable digital filter at the DSP $[7,20]$. Unlike the case of DD, which is severely affected by $\mathrm{CD}$, especially when SSB is not adopted, with a CO-Rx, thanks to the digital compensation, the VCSEL chirp interplay with $\mathrm{CD}$ can be neglected $[7,17,20]$. Then, the frequency difference between the local oscillator (LO) and the received signal is estimated. This value is then used to drive the LO to compensate for the laser frequency drift and obtain an accurate baseband representation of the received signal. A digital lowpass filter (LPF) follows, and the square modulus function is applied to obtain a representation of the received signal power. Note that the filter is specifically designed to avoid unwanted mixing products between the $\mathrm{LO}$ and the received signal itself, due to nonideal components, while rejecting noise. Therefore, its response is expected to feature sharp edges and a rather limited bandwidth. This also allows the easy retrieval of the signal of interest over the adjacent channels [20]. The LO needs to be 
able to provide an accurate frequency difference between LO and received signals.

It is worth noting that, considering a VCSEL linewidth of $5 \mathrm{MHz}$, it has been numerically shown that the LO linewidth, up to a value of $1 \mathrm{MHz}$, has a limited impact on the system performance, and a possible frequency mismatch with respect to the signal central frequency causes a modest impairment [20].

\section{EXPERIMENTAL ASSESSMENT}

In view of assessing the proposed photonic system architecture to target the dynamic 5G-supportive MAN as described in Section 2, we have emulated multihop connections across multiple HLs in a real testbed network, the ADRENALINE testbed $[11,21]$.

To experimentally evaluate the performance of the proposed photonic system architecture, a single flow connection is considered over up to six hops and $160 \mathrm{~km}$. It is enabled by an S-BVT adopting a VCSEL with $10 \mathrm{GHz}$ bandwidth. The path includes the real testbed network and $25 \mathrm{~km}$ 19-core MCF. Multiple switching nodes with different functionalities according to the $\mathrm{HL}$ and photonic system architecture are considered. Specifically, it is considered an HL4 node based on AWGs and up to five HL3 and HL2/1 nodes, including all the functionalities described in Section 3.

\section{A. Experimental Setup}

The experimental setup is described in Figs. 6 and 7. At the $S$-BVT Tx, a single flow is generated enabling a single-mode VERTILAS VCSEL with $10 \mathrm{GHz}$ bandwidth. It operates at $1545.32 \mathrm{~nm}(194.000 \mathrm{THz})$, which corresponds to a nominal central frequency $(n)$ of 144 according to [22] for flexi-grid and represents VCSEL number 6, within subMOD 2 of SOI-chip fundamental MOD 1, identified by the tuple VCSEL-6/subMOD-2/MOD-1 (see Fig. 6 inset).

At the S-BVT Tx, the adaptive DSP based on OFDM enabling adaptive loading, described in Section 3.B and reported in Fig. 5, is used with a $64 \mathrm{GSa} / \mathrm{s}$ DAC for directly modulating the VCSEL. For the S-BVT Rx, we consider both DD and CO-Rx module options.

The former is a PIN diode with $20 \mathrm{GHz}$ bandwidth and includes a transimpedance amplifier (TIA); the latter has

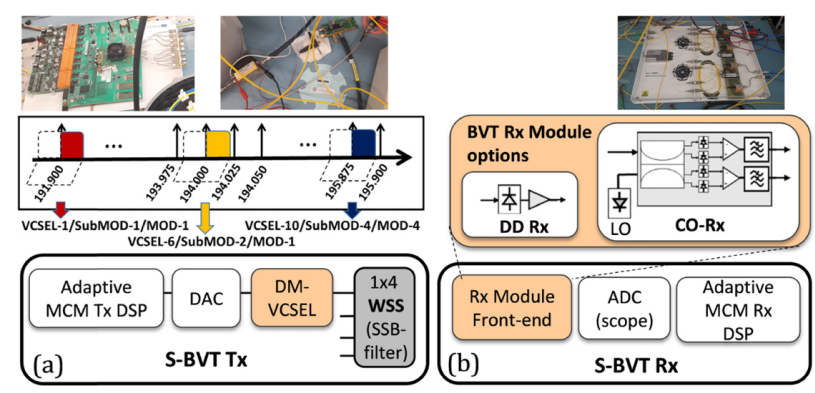

Fig. 6. S-BVT adopted in the experiments. (a) Tx based on a DMVCSEL operating at $194.000 \mathrm{THz}$, corresponding to VCSEL 6 of subMOD 2 of MOD 1 (in the inset pictures, DAC and VERTILAS VCSEL with a driver and evaluation board); (b) S-BVT Rx with $\mathrm{DD}$ and CO-Rx options (in the inset picture, a CO-Rx with discrete components).

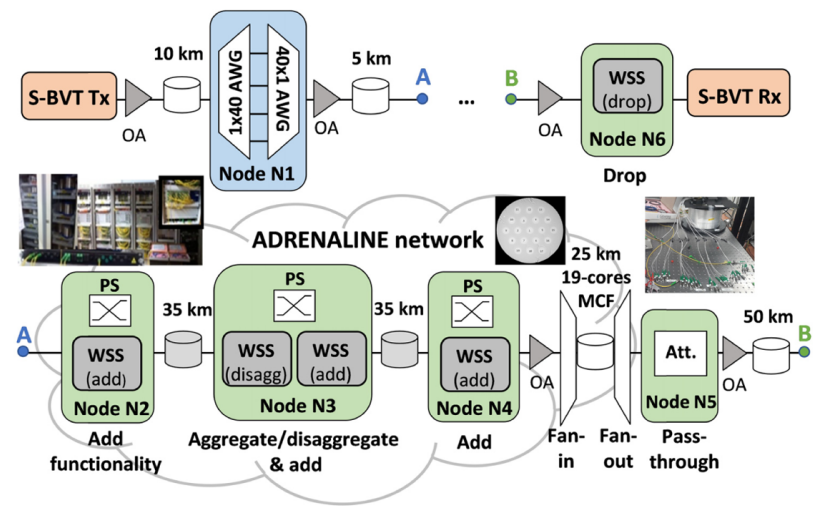

Fig. 7. Schematic of the transmission system. Case of $15 \mathrm{~km}$ HL4-HL3 connection (point A coincides with point B) and case of $160 \mathrm{~km}$ HL4-HL2/1 connection with multiple hops including the ADRENALINE testbed network path and $25 \mathrm{~km}$ 19-core MCF.

discrete components [see the inset picture of Fig. 6(b)] with up to $40 \mathrm{GHz}$ bandwidth and includes a tunable LO. The received signal is captured by an oscilloscope (at $50 \mathrm{GSa} / \mathrm{s}$ ) acting as ADC and postprocessed by the adaptive DSP, as detailed in Section 3.B. In the case of a CO-Rx, a full-featured DSP, including the blocks for $I$ and $Q$ component recovery (indicated in Fig. 5 with the dashed line and detailed in the bottom inset), is used.

We have considered a multihop path from HL4 to HL2/1 with six hops covering a total of $160 \mathrm{~km}$ and traversing up to six WSSs and two 100-GHz AWGs. The WSS bandwidth has been set to $50 \mathrm{GHz}$. The path includes three nodes of the ADRENALINE testbed network, two standard single-mode fiber (SSMF) links of $35 \mathrm{~km}$, and $25 \mathrm{~km} \mathrm{MCF}$ with fan-in and fan-out, in addition to SSMF spools of 10, 5 , and $50 \mathrm{~km}$. The links within the ADRENALINE network are preamplified by means of erbium-doped fiber amplifiers (EDFAs); the rest of the setup optical amplifiers (OAs) are indicated in Fig. 7. The WSS at the Tx is for SSB filtering [as in the schematic of Fig. 6(a)], enabling a more robust transmission. The first node (N1) represents an HL4 node and adopts 100-GHz AWGs to forward traffic from the S-BVT towards the upper HL nodes. According to the envisioned node architecture for HL3 and HL2/1, described in Section 3, the WSS-based filtering stages at node N2 implement the add functionality of an HL3 node, while the subsequent node includes an additional WSS to take into account the aggregate/disaggregate functionality. This allows the considered flow to be spectrally combined with other flows at the HL3 node N3. Then, node N4 performs the add functionality at HL2/1. It is worth noting that at the ADRENALINE nodes (N2, N3, and N4), variable and fixed attenuators have been considered in the setup to emulate the PS modules present at the HL3 and HL2/1 nodes. After the 19-core MCF, node N5 emulates the pass-through functionality at HL2/1 of the polymer PS module adopting a $2-\mathrm{dB}$ attenuator (Att. in Fig. 7) [8,15]. In the last node (N6), a WSS is considered for dropping the traffic towards the S-BVT Rx.

In the case of considering IP off-loading, the path of interest to be analyzed is the entire path depicted in Fig. 7, where the 
S-BVT Tx and Rx are illustrated in Figs. 6(a) and 6(b), respectively. To analyze a shorter HL4-HL3 connection, points A and B of Fig. 7 are shortcut. Thus, in the first case, N6 represents an HL2/1 node, while in the second case, it represents an HL3 node.

\section{B. Experimental Results}

In this subsection, we report the performance of the VCSEL-based S-BVT of Fig. 6 adopting either DD or a CO-Rx.

At the offline DSP, we consider an OFDM signal with 512 subcarriers over $16 \mathrm{GHz}$. UL is used to estimate the SNR and the RA BL algorithm is applied for maximizing the capacity performance (Section 3.B). The target bit error ratio (BER) for a successful transmission is set to $4.62 \cdot 10^{-3}$, considering hard-decision forward error correction (HD-FEC) with 7\% overhead [11].

Figure 8 shows the capacity performance for the back-toback (B2B) case at the varying of the optical SNR (OSNR). The reference bandwidth considered for the OSNR measurement/analysis is $12.5 \mathrm{GHz}(0.1 \mathrm{~nm})$. The maximum capacity achieved with DD is $36.9 \mathrm{~Gb} / \mathrm{s}$, while with a CO-Rx it is $31.2 \mathrm{~Gb} / \mathrm{s}$. We have verified that this is due to the adopted CO-Rx module, which is implemented with discrete components. In fact, using a commercial integrated CO-Rx with similar responsivity, a capacity of $34.6 \mathrm{~Gb} / \mathrm{s}$ is supported at the OSNR value of $33.4 \mathrm{~dB}$, close to the value supported by $\mathrm{DD}$ $(32.8 \mathrm{~Gb} / \mathrm{s})$ and higher than the one obtained by adopting a discrete CO-Rx $(27.6 \mathrm{~Gb} / \mathrm{s})$. At the OSNR value of $26.7 \mathrm{~dB}$, corresponding to a capacity of $23.7 \mathrm{~Gb} / \mathrm{s}$, the DD and CO-Rx $\mathrm{B} 2 \mathrm{~B}$ curves cross, and for lower OSNR values, it is shown that the CO-Rx (even implemented with discrete components) outperforms DD [11]. Then, we have analyzed the multihop connections as described in Section 4.A. The obtained results are summarized in Table 2.

For the HL4-HL3 connection, including only N1 and N6 nodes (path shortcutting points A and B in Fig. 7), the capacity performance of both DD and the CO-Rx is slightly affected, with similar reduction with respect to the $\mathrm{B} 2 \mathrm{~B}$ case at the same OSNR values. In the case of HL4-HL2/1 connection, including multiple hops, the testbed network and the MCF (entire

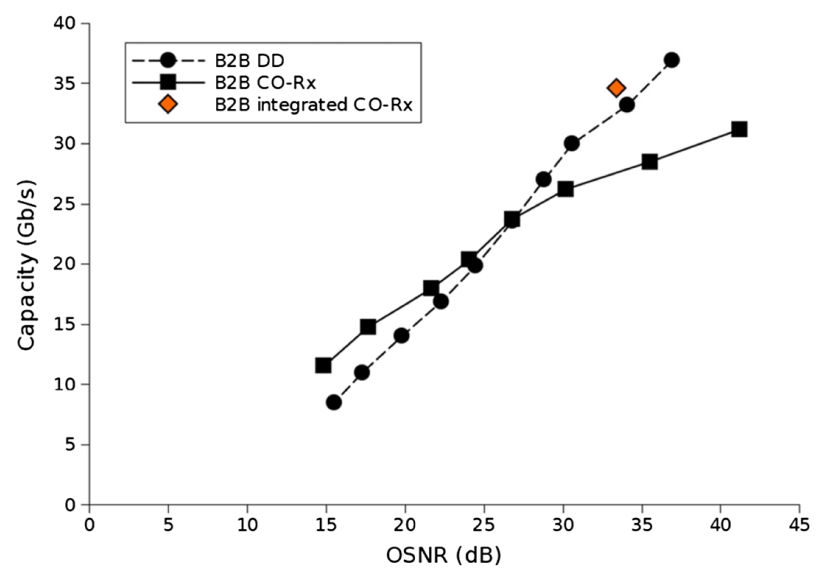

Fig. 8. $\quad \mathrm{B} 2 \mathrm{~B}$ performance adopting $\mathrm{DD}$, a discrete or integrated CO-Rx.
Table 2. Connectivity Performance Adopting DD and the CO-Rx Module at the VCSEL-Based S-BVT

\begin{tabular}{lcccccc}
\hline & \multicolumn{3}{c}{ Path } & & \multicolumn{2}{c}{ Capacity $(\mathbf{G b} / \mathbf{s})$} \\
\cline { 2 - 4 } \cline { 6 - 7 } HL Connection & Hop \# & $\mathbf{~ k m}$ & OSNR (dB) & & DD & CO-Rx \\
\hline HL4-HL3 & 2 & 15 & 35.7 & & 33.7 & 26.4 \\
HL4-HL2/1 & 6 & 160 & 26.4 & & 7.6 & 22.6 \\
\hline
\end{tabular}

path of Fig. 7), improved performance (similar to the B2B case at the same OSNR values) can be obtained with the CO-Rx DSP. This is thanks to the CO-Rx DSP module allowing compensation for the $\mathrm{CD}$, which in turn severely affects the $\mathrm{DD}$ scheme.

\section{PHOTONIC SYSTEM ARCHITECTURE MODELING AND ASSESSMENT TOWARDS MULTI-TB/S CONNECTIVITY}

In the previous section, an S-BVT based on a VCSEL with a limited electro-optical bandwidth of $10 \mathrm{GHz}$ was considered, combined with both DD and a CO-Rx. Adopting a higher bandwidth VCSEL and an integrated CO-Rx, it is envisioned to improve the achievable capacity performance to target up to $50 \mathrm{~Gb} / \mathrm{s}$ per flow. This enables each module to support up to $2 \mathrm{~Tb} / \mathrm{s}$ and target multi- $\mathrm{Tb} / \mathrm{s}$ connectivity over a dynamic 5G-supportive MAN. Thus, based on the obtained experimental results, we extract a simulation model to estimate the performance in the case of considering an improved photonic system, as described in Section 3, including an optimized SC-VCSEL with a bandwidth of $18 \mathrm{GHz}$ and an integrated CO-Rx.

First, the developed simulative tool [17] has been validated by modeling the experimental results reported in the previous section. This numerical model is then compared with a theoretical model [23] developed for the proposed photonic system architecture. Thereafter, simulations have been performed to estimate the performance of the photonic systems for the targeted HL4-HL2/1 connection considering a higher bandwidth VCSEL and an integrated CO-Rx.

In particular, the model of a $45 \mathrm{GHz}$ OM4245 Tektronix coherent detector has been used [24]. Due to the multiple hops, the effect of filtering can severely impact the transmission performance, so the presence of the cascade of either $50-\mathrm{GHz}$ or $25-\mathrm{GHz}$ WSS filters has been considered. Table 3 reports the main parameters of the modeling and simulative assessment.

Specifically, the CO-Rx exploits a $14.5 \mathrm{dBm} \mathrm{LO}$ with $300 \mathrm{kHz}$ linewidth. The DM VCSEL is modeled considering both the intrinsic modulation properties and the extrinsic device parasitic components, following the approach reported in [25], leading to the overall electrical modulation frequency responses reported in Table 3. The impact of optical filtering is evaluated using the experimentally measured $50 \mathrm{GHz}$ and $25 \mathrm{GHz}$ WSS transfer functions [26] generated by a Finisar Waveshaper programmable filter. The considered propagation medium is an SSMF with $0.25 \mathrm{~dB} / \mathrm{km}$ attenuation and $16 \mathrm{ps} / \mathrm{nm} \mathrm{km}$ dispersion at $1545 \mathrm{~nm}$. The OSNR at the Rx is varied according to the measurements in order to fit the experimental data and to estimate the performance of the 
Table 3. Parameters Used for Modeling and Simulations (Numerical Analysis) with an Improved Photonic System

\begin{tabular}{lcc}
\hline & Modeling & Simulations \\
\hline VCSEL bandwidth & $10 \mathrm{GHz}$ & $18 \mathrm{GHz}$ \\
Modulation bandwidth & $16 \mathrm{GHz}$ & $20 \mathrm{GHz}$ \\
MCM subcarrier \# & 512 & 512 \\
MCM subcarrier spacing & $31.25 \mathrm{MHz}$ & $39.06 \mathrm{MHz}$ \\
Rx bandwidth & $20 \mathrm{GHz}$ & $45 \mathrm{GHz}$ \\
AWG bandwidth & $100 \mathrm{GHz}$ & $100 \mathrm{GHz}$ \\
WSS bandwidth & $50 \mathrm{GHz}$ & $50 \mathrm{GHz}, 25 \mathrm{GHz}$ \\
Path length & $0,15,160 \mathrm{~km}$ & $0,160 \mathrm{~km}$ \\
\hline
\end{tabular}

photonic system for different OSNR conditions. The SSB transmission is performed by properly detuning the first WSS transfer function with respect to the carrier of the MCM signal. Similar DSP blocks to those described in Section 3 are applied, performing a suboptimal adaptive $\mathrm{BL}$ algorithm [27] and considering the same target BER of $4.62 \cdot 10^{-3}$ (with a 7\% overhead HD-FEC limit) for single-channel and single-polarization transmission.

\section{A. Numerical and Theoretical Modeling Analysis}

The modeling of the experimental data has been performed according to the parameters indicated in Table 3 to adapt the simulation tools to the system considered in the experimental setup. As reported in Fig. 9, a very good fitting of the measured capacity as a function of the OSNR in the case of DD can be achieved in the $\mathrm{B} 2 \mathrm{~B}$ condition.

In order to show the capabilities of adopting a fully integrated CO-Rx, a CO-Rx showing the same performance as DD in terms of the electrical SNR has been simulated. Accordingly, it has been found that the $\mathrm{B} 2 \mathrm{~B}$ performance in the case of using an integrated CO-Rx allows similar results as DD to be obtained, also in line with the experimental verification obtained in the lab with such a type of CO-Rx (orange diamond point in Fig. 8). Also, a simplified mathematical modeling has been developed that can be understood as a

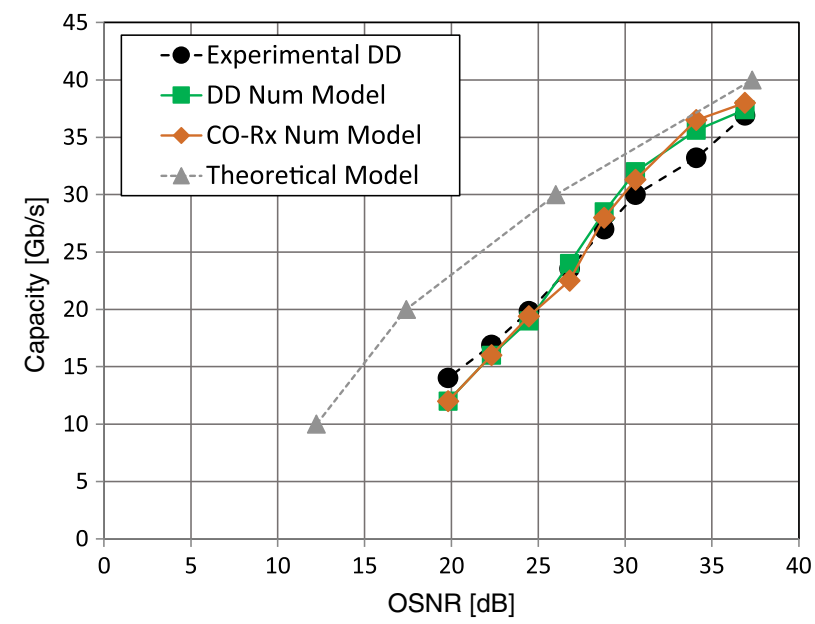

Fig. 9. $\mathrm{B} 2 \mathrm{~B}$ performance. $\mathrm{DD}$ experimental curve as in Fig. 8 (OSNR range 20-37 dB), numerical model assessment for DD and an integrated CO-Rx, and the CO-Rx theoretical model. bound limit to the simulation results. In this case, a simple model can be used for the proposed CO-Rx module [23]. The SNR of each OFDM symbol after demodulation can be expressed as

$$
\mathrm{SNR}=\frac{\left(R^{2} P_{s 0} P_{l}\right)^{2}}{2 \sigma^{4}+4 \sigma^{2}\left(R^{2} P_{s a} P_{l}\right)^{2}},
$$

$R$ being the responsivity of the photodetectors, $P_{s 0}$ the amplitude of the OFDM signal, $P_{s a}$ the average received power, $P_{l}$ the power of the $\mathrm{LO}$, and

$$
\sigma^{2}=\frac{R^{2} P_{s a} P_{l} B_{e}}{2 \mathrm{OSNR} B_{o}}
$$

where $B_{o}$ is the reference bandwidth for the OSNR, e.g., $12.5 \mathrm{GHz}$, and $B_{e}$ is the single-sided electrical bandwidth of the MCM signal.

Combining Eqs. (1) and (2) with well-known expressions, the probability of bit error of different symbol constellations can be derived [28]. Therefore, we can easily obtain the OSNR values required for a $4.62 \cdot 10^{-3} \mathrm{BER}$ at different constellations (i.e., different capacities). Results shown in Fig. 9 correspond to the parameters of Table 3 and assume $P_{s a} / P_{s 0}=9.2 \mathrm{~dB}$. There, we can observe that the model is in good agreement with the data. In fact, for high OSNR values (when the electrical SNR is high) the model matches the simulations quite well ( $\sim 1 \mathrm{~dB}$ difference). Nevertheless, when the OSNR decreases, the model differs from the gathered data $(>3-5 \mathrm{~dB})$. This is due to the fact that the numerical analysis includes other effects not considered in the mathematical model. Actually, the model is based on ideal intensity modulation, and it does not take into account optical SSB filtering, laser chirp, and parasitic effects. Furthermore, according to [23], the model assumes Gaussian approximation for a noncentral chi-square process associated with the noise. Therefore, the model is more accurate for higher than lower OSNR values.

The developed simulation tool also permits one to properly approximate the impact of SSMF propagation, leading to the correct estimation of the performance after $15 \mathrm{~km}$ and $160 \mathrm{~km}$ connections. For the HL4-HL3 connection, similar performance as in B2B has been obtained, adopting either $\mathrm{DD}(36.5 \mathrm{~Gb} / \mathrm{s}$ at a $35.7 \mathrm{~dB}$ OSNR) or an integrated CO-Rx (36.7 Gb/s at a $35.7 \mathrm{~dB}$ OSNR). As reported in Section 4, the drastic reduction of the achievable capacity for the case of HL4-HL2/1 connection is due to CD, which cannot be compensated for in standard DD systems. In the case of a CO-Rx, this effect can be efficiently compensated for at the Rx DSP, leading to improved performance, as experimentally demonstrated in Section 4 (Table 2). This has also been numerically verified with simulations, obtaining $8.5 \mathrm{~Gb} / \mathrm{s}$ and $23.3 \mathrm{~Gb} / \mathrm{s}$ (at the OSNR value of $26.4 \mathrm{~dB}$ ) in the cases of $\mathrm{DD}$ and a CO-Rx, respectively.

\section{B. Expected Performance Adopting the Target Photonic System}

The simulation tool has been exploited to estimate the performance in the case of adopting a photonic system with a 


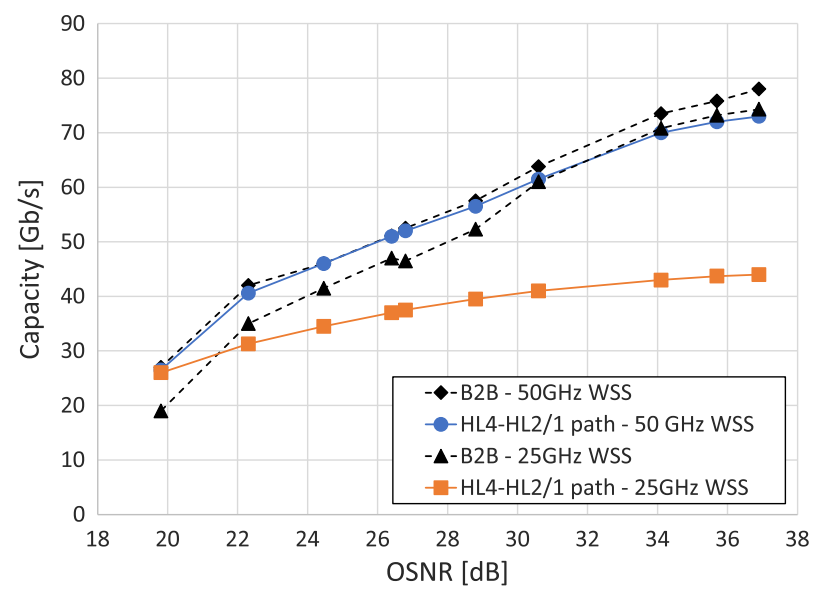

Fig. 10. B2B and HL4-HL2/1 path (160 km SSMF) with $50-\mathrm{GHz}$ and $25-\mathrm{GHz}$ WSS filtering.

higher bandwidth VCSEL and an integrated CO-Rx, according to the target solution. Furthermore, the supported capacity has been numerically analyzed, considering both $50 \mathrm{GHz}$ and $25 \mathrm{GHz}$ granularity for the WSS-based nodes both in the B2B condition and after the entire $160 \mathrm{~km}$ connection. Results are reported in Fig. 10. In the B2B condition, two WSSs are taken into account (including SSB and drop filtering at the Tx and $\mathrm{Rx}$, respectively), while in the case of $160 \mathrm{~km}$ (SSMF is considered) the optical signal traverses two 100-GHz AWGs and six WSSs, as in the experimental setup of Fig. 7.

Since the electro-optical bandwidth of the DM VCSEL is almost doubled, a strong increase in the achievable capacity can be noticed. In the case of adopting $50-\mathrm{GHz}$ WSSs, the target $50 \mathrm{~Gb} / \mathrm{s}$ capacity can be supported for OSNR values above $26 \mathrm{~dB}$ (and beyond $40 \mathrm{~Gb} / \mathrm{s}$ above a $22 \mathrm{~dB}$ OSNR). The maximum capacity achieved in B2B at a $36.9 \mathrm{~dB}$ OSNR is $78 \mathrm{~Gb} / \mathrm{s}$, while at the minimum considered OSNR value of $19.8 \mathrm{~dB}$ the capacity decreases to about half of the target, being $27 \mathrm{~Gb} / \mathrm{s}$. For the $160 \mathrm{~km} \mathrm{SSMF}$ path, no significant reduction of the supported capacity with respect to B2B arises for an OSNR lower than $28 \mathrm{~dB}$, in line with the obtained experimental results. A lowering of the supported capacity can be appreciated for an OSNR beyond $30 \mathrm{~dB}$, where the presence of the WSS cascade slightly limits the high-frequency subcarriers BL. In the case of considering $25-\mathrm{GHz}$ WSS-based nodes, a reduction of the B2B capacity with respect to the $50 \mathrm{GHz}$ granularity is observed, owing to a slight reduction of the available optical spectrum to be loaded by the MCM subcarriers. The maximum capacity achieved in B2B at a $36.9 \mathrm{~dB}$ OSNR is $74.3 \mathrm{~Gb} / \mathrm{s}$. A very strong limitation arises in the $160 \mathrm{~km}$ path connection, due to the filter narrowing effect of the WSS-based node cascade with respect to the B2B. Actually, the superposition of $25-\mathrm{GHz}$ WSSs at the nodes including point $\mathrm{A}$ and $\mathrm{B}$ of Fig. 7 leads to an equivalent filter characterized by an effective bandwidth of about $14.5 \mathrm{GHz}$ (lower than the VCSEL bandwidth), while for $50-\mathrm{GHz}$ WSSs, an equivalent filter with $29 \mathrm{GHz}$ bandwidth is obtained $[26,29]$. Thus, in the case of $25 \mathrm{GHz}$ granularity, the limitation on the available spectral resource reduces the supported capacity below $45 \mathrm{~Gb} / \mathrm{s}$, even at high OSNR values. Nevertheless, for all the analyzed cases,
$30 \mathrm{~Gb} / \mathrm{s}$ can be supported for an OSNR higher than $22 \mathrm{~dB}$. Thus, up to $1.2 \mathrm{~Tb} / \mathrm{s}$ can be achieved enabling $40 \mathrm{VCSEL}$ flows at this rate.

The impact of propagation and filtering here analyzed over the targeted path shows a different impact of the cascading of several WSS filters at the two different analyzed granularities: as long as the filter narrowing effect does not induce a significant reduction of the available spectrum for $\mathrm{BL}$, the capacity is mainly affected by the OSNR variation; otherwise, the capacity decrease does not follow a linear evolution with the OSNR, being mainly affected by the filtering operation. As expected, a fine granularity of $25 \mathrm{GHz}$ is more critical $[9,29]$. However, the impact of filtering (on the achievable capacity at the varying of the node number), for the proposed approach at this granularity, can be eventually mitigated with a slight reduction of the electrical spectrum, by means of a suitable selection of the electrical subcarriers to be modulated [20]. This can also be beneficial in the presence of adjacent channels and considering the actual VCSEL chirp coefficients (inducing the spectral broadening) $[17,20]$. Thus, thanks to the adoption of MCM, with a suitable selection of the modulation bandwidth/amplitude and filtering at the DSP level, the cross talk can be mitigated and the performance optimized. Indeed, nonlinear effects should be further investigated (preliminary results have been obtained in the framework of [13]), since they can affect the performance limiting the target capacity and the scalability of the proposed solution, especially at the increasing of the path length [30].

\section{PROGRAMMABILITY}

Dynamic flexi-grid optical connection requests specifying the source, destination, and required capacity (in b/s) are automatically received, processed, and served by a centralized SDN controller. For each incoming request, the SDN controller computes and selects the spatial (i.e., node and links) and spectral resources (i.e., frequency slot, FS) between the connection end points fulfilling the capacity demands. Additionally, capabilities and features derived from the underlying data plane infrastructure, such as the optical switch filtering, the maximum end-to-end path distance, as well as the spectrum continuity and contiguity constraints, are considered when computing a feasible route. Thereby, the routing process handled within the SDN controller uses as input the connection demands along with an updated view of the transport network entailing (i) the availability of the source S-BVT Tx (i.e., an unused VCSEL and supported central frequency), (ii) the availability of the destination S-BVT Rx (i.e., an unused CO-Rx), and (iii) the available optical spectrum on every network link. This input information is then used by the routing mechanism, which is referred to as the routing and spectrum assignment (RSA) algorithm. In [31], an online RSA algorithm tailored to the specific characteristics of network elements, such as the VCSEL-based and Co-Rx S-BVTs, along with the use of different optical switching nodes sorted by HL (i.e., HL4, HL3, and HL2/1) is presented.

The output of a successful RSA computation for a given request provides (i) the selected VCSELs (and thus the associated central frequencies for the whole connection FS) at the 
source node, (ii) the LO to be tuned at the destination CO-Rx, and (iii) the nodes and links to be traversed and the respective FS. Herein, the FS is determined according to the ITU-T G.694.1 recommendation [22]. Specifically, the FS is formed by (i) a nominal central frequency $(n)$ in terahertz, computed using the expression $193.100 \mathrm{THz}+n * 0.00625 \mathrm{THz}(n$ being either a positive or negative integer) and (ii) a slot width $(m)$ in gigahertz, obtained according to the expression $6.25 * m$. It is worth outlining that, since heterogenous optical switches supporting different filtering capabilities can be traversed, the resulting FS (i.e., $n$ and $m$ ) to be configured on each node needs to fulfill such filtering capabilities. In other words, HL4 nodes supporting $100 \mathrm{GHz}$ filters require that the FS $m$ parameter is set to 8 . On the other hand, for the HL3 and HL2/1 using $50 \mathrm{GHz}$ filters, the FS $m$ is 4 . For the sake of completeness, the FS $n$ parameter is determined by the selected S-BVT VCSEL. In the considered experimental transport setup, the S-BVT Tx element operates at $194.000 \mathrm{THz}$, which corresponds to $n=144$.

The programmability of the involved network nodes and devices (i.e., an S-BVT Tx VCSEL and a CO-Rx) to accommodate a computed optical connection is commanded by the SDN controller directly communicating with the corresponding network agents. To this end, a RESTful API described and validated in [32] is used. This API creates client-server relationships between the SDN controller and the agents to enable (i) the retrieval of the updated status of the network resources (e.g., a used/available S-BVT Tx VCSEL and a CO-Rx) and (ii) the allocation and deallocation of the selected resources (i.e., S-BVT VCSELs, a CO-Rx LO, optical spectrum links).

Figure 11 depicts the logical workflow (via the defined RESTful API [31]) used for configuring the network elements and devices specified in the RSA output. It basically encompasses two steps: (a) programming the S-BVT Tx and Rx at the source and destination end points and (b) programming the FS for each input and output pair constituting the cross connection at each traversed (HL) node. For both the S-BVT Tx and $\mathrm{Rx}$, two dedicated POST messages carry the selected central frequency (i.e., $n=144$ ) that allow the determination of the S-BVT Tx VCSEL to be allocated and the LO to be tuned
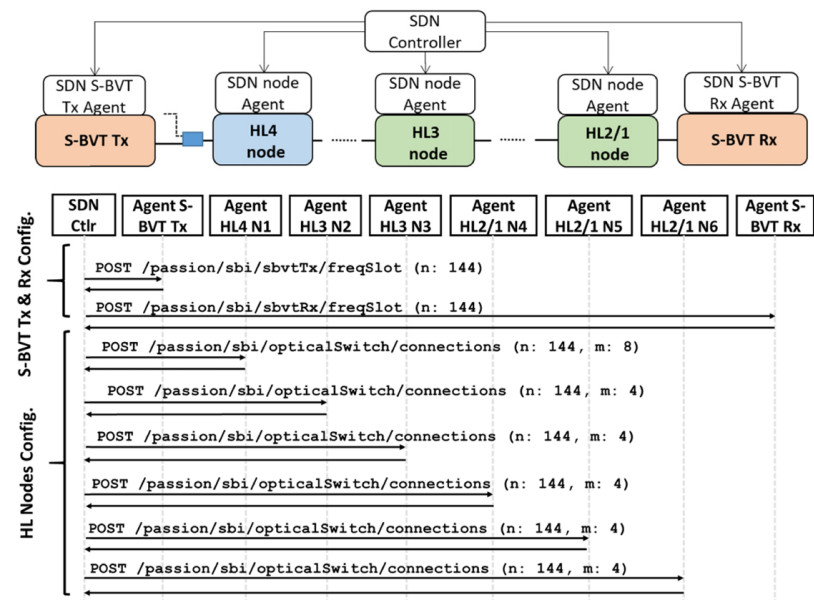

Fig. 11. RESTful API logical workflow for programming the transport network elements and devices.

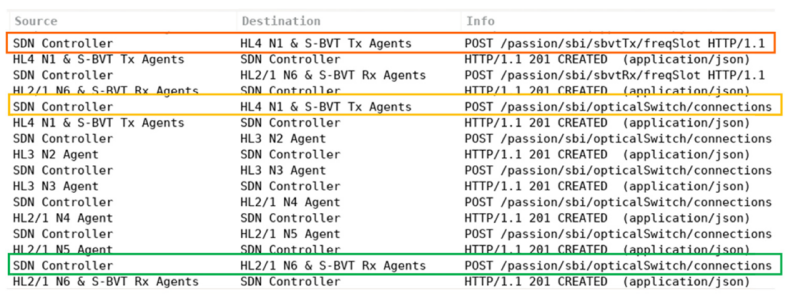

(a)

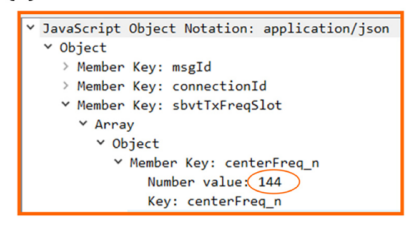

(b)

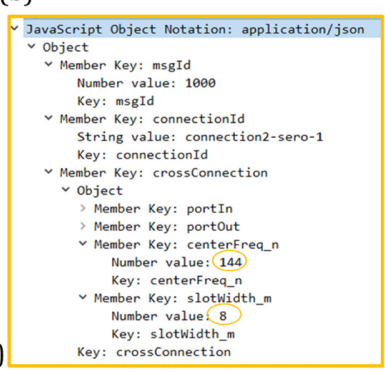

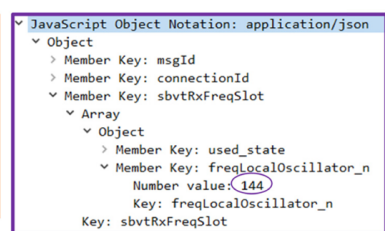

(c)

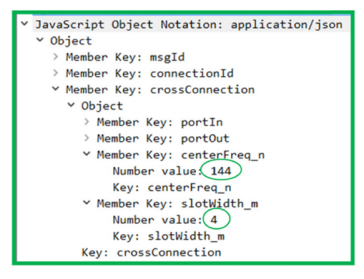

(e)
Fig. 12. (a) Captured RESTful API control messages; (b) JSON contents for S-BVT Tx; (c) JSON contents for S-BVT Rx; (d) JSON contents for HL4 node; (e) JSON contents for HL3 and HL2/1 nodes.

at the S-BVT Rx, respectively. For each HL optical switch, a POST message yields the information about the FS to be used. That is, $n=144$, while $m$ is set to either 4 or 8 , depending on the HL4 or HL3 and HL2/1 node type. Figure 12(a) illustrates, via a protocol analyzer, a screenshot of the set of RESTful API messages between the SDN controller and the agents validating the resulting data plane programmability for setting up the optical flow. Additionally, Figs. 12(b)-12(e) provide the JSON-encoded information describing the required parameters to configure the S-BVT Tx (i.e., VCSEL selection), S-BVT Rx (i.e., a LO), and the HL4 and HL2/1 configuration detailing the input and output port identifiers and the FS (i.e., $n$ and $m$ ).

\section{CONCLUSIONS}

We have described and assessed a modular SDN-enabled photonic system architecture designed to address the challenges of 5G-supportive MANs. In the experimental validation, an S-BVT based on VCSEL technology with $10 \mathrm{GHz}$ bandwidth is considered, and DD and CO-Rx modules are compared in a testbed network. It is shown that, while DD is more affected by OSNR degradation and path length increase, a $\mathrm{CO}-\mathrm{Rx}$, even using discrete components, allows the support of $>30 \mathrm{~Gb} / \mathrm{s}$ in $\mathrm{B} 2 \mathrm{~B}$ and $>20 \mathrm{~Gb} / \mathrm{s}$ capacity, over a $160 \mathrm{~km}$ multihop path, including six aggregation nodes and 19-core MCF. By adopting programmable (SDN-enabled) photonic transceivers based on $\geq 18 \mathrm{GHz}$ VCSELs and an integrated CO-Rx, better performance is expected to be achieved. In fact, the results, obtained with the derived numerical model, 
show that with $50 \mathrm{GHz}$ granularity, the target $50 \mathrm{~Gb} / \mathrm{s}$ capacity per flow can be supported over the six-hop $160 \mathrm{~km}$ (SSMF) HL4-HL2/ 1 connection for OSNR values above $26 \mathrm{~dB}$. When the granularity is $25 \mathrm{GHz}$, the filter narrowing effect could severely impact the performance, thus limiting the available bandwidth. Nevertheless, $1.2 \mathrm{~Tb} / \mathrm{s}$ capacity can be achieved when the modular S-BVT is configured to enable 40 VCSELs.

Thanks to the adoption of MCM with CO-Rx, with a suitable selection of the modulation bandwidth/amplitude and specific filtering at the DSP level, the cross talk can be mitigated and the performance optimized to increase the supported capacity. As a next step, the nonlinear effects will be investigated to further assess the target capacity and scalability of the proposed solution, especially as the path length is increased. Suitable path selection could be particularly relevant to address this issue. As future work, the agility of the S-BVT, based on the described technologies, in terms of $T x / R x$ configuration/setup time will be investigated within the real testbed network.

Funding. European Commission [EU H2020 PASSION Project (GA 780326)]; Ministerio de Ciencia, Innovación y Universidades [AURORAS project (RTI2018-099178-BI00)].

\section{REFERENCES}

1. "Cisco Visual Networking Index: Forecast and Trends, 2017-2022," 2019, https://www.davidellis.ca/wp-content/uploads/2019/05/ cisco-vni-feb2019.pdf.

2. M. S. Moreolo, J. M. Fabrega, L. Nadal, R. Martínez, and R. Casellas, "Synergy of photonic technologies and software-defined networking in the hyperconnectivity era," J. Lightwave Technol. 37, 3902-3910 (2019).

3. M. Mueller, W. Hofmann, T. Grundl, M. Horn, P. Wolf, R. D. Nagel, E. Ronneberg, G. Bohm, D. Bimberg, and M.-C. Amann, "1550 nm highspeed short-cavity VCSELs," IEEE J. Sel. Top. Quantum Electron. 17, 1158-1166 (2011).

4. S. Bhat, M. Harjanne, F. Sun, M. Cerchi, M. Kapulainen, A. Hokkanen, G. Delrosso, and T. Alto, "Low loss devices fabricated on the open access $3 \mu \mathrm{m}$ SOI waveguide platform at VTT," European Conference on Integrated Optics, Ghent, Belgium, 2019, paper T.Po2.

5. M. S. Moreolo, J. M. Fabrega, L. Nadal, F. J. Vilchez, A. Mayoral, R. Vilalta, R. Muñoz, R. Casellas, R. Martínez, M. Nishihara, T. Tanaka, T. Takahara, J. C. Rasmussen, C. Kottke, M. Schlosser, R. Freund, F. Meng, S. Yan, G. Zervas, D. Simeonidou, Y. Yoshida, and K. Kitayama, "SDN-enabled sliceable BVT based on multicarrier technology for multi-flow rate/distance and grid adaptation," J. Lightwave Technol. 34, 1516-1522 (2016).

6. D. Larrabeiti, G. Otero-Pérez, J. A. Hernández, P. Reviriego, J. Fernández-Palacios, V. López, M. S. Moreolo, R. Martínez, and J. M. Fabrega, "Tradeoffs in optical packet and circuit transport of fronthaul traffic: the time for SBVTs?" in Optical Network Design and Modelling (ONDM) (2020).

7. M. S. Moreolo, L. Nadal, J. M. Fabrega, F. J. Vilchez, C. Neumeyr, A. Gatto, P. Parolari, and P. Boffi, "VCSEL-based sliceable bandwidth/bitrate variable transceivers," Proc. SPIE 10946, 1094606 (2019).

8. M. S. Moreolo, R. Martínez, L. Nadal, J. M. Fabrega, N. Tessema, N. Calabretta, R. Stabile, P. Parolari, A. Gatto, P. Boffi, G. Otero, D. Larrabeiti, J. A. Hernandez, P. Reviriego, J. P. Fernández-Palacios, V. López, G. Delrosso, C. Neumeyr, K. Solis-Trapala, G. Parladori, and G. Gasparini, "Spectrum/space switching and multi-terabit transmission in agile optical metro networks," in OptoElectronics and Communications Conference/International Conference on Photonics in Switching and Computing (OECC/PSC) (2019).

9. L. Nadal, J. M. Fabrega, M. S. Moreolo, R. Casellas, R. Muñoz, L. Rodríguez-Navas, R. Vilalta, F. J. Vilchez, and R. Martínez, "SDNenabled sliceable transceivers in disaggregated optical networks," J. Lightwave Technol. 37, 6054-6062 (2019).

10. L. Nadal, M. S. Moreolo, J. Alberto Hernández, J. M. Fabrega, R. Casellas, R. Muñoz, R. Vilalta, L. Rodríguez-Navas, F. J. Vilchez, and R. Martínez, "SDN-enabled S-BVT for disaggregated networks: design, implementation and cost analysis," J. Lightwave Technol. 38, 3037-3043 (2020).

11. M. S. Moreolo, J. M. Fabrega, L. Nadal, R. Martínez, R. Casellas, J. Vílchez, R. Muñoz, R. Vilalta, A. Gatto, P. Parolari, P. Boffi, C. Neumeyr, D. Larrabeiti, G. Otero, and J. P. Fernández-Palacios, "Experimental assessment of a programmable VCSEL-based photonic system architecture over a multi-hop path with 19core MCF for future agile Tb/s metro networks," in Optical Fiber Communication Conference (2020), paper M4D.5.

12. D. Larrabeiti, J. Fernández-Palacios, G. Otero, M. S. Moreolo, J. M. Fabrega, R. Martínez, P. Reviriego, and V. López, "All-optical paths across multiple hierarchical levels in large metropolitan area networks," in Asia Communications and Photonics Conference (ACP) (2019), paper M4A.206.

13. http://www.passion-project.eu/.

14. J. M. Fabrega, M. S. Moreolo, L. Martin, A. Chiadò Piat, E. Riccardi, D. Roccato, N. Sambo, F. Cugini, L. Potì, S. Yan, E. Hugues-Salas, and D. Simeonidou, "On the filter narrowing issues in elastic optical networks," J. Opt. Commun. Netw. 8, A23-A33 (2016).

15. H.-D. Jung, "Polymer-based optical switch for future metro-area networks," in OSA Advanced Photonics Congress (2020).

16. P. Boffi, P. Parolari, A. Gatto, M. Rapisarda, M. S. Moreolo, L. Nadal, J. M. Fabreag, N. Calabretta, R. Stabile, N. Tessema, D. Larrabeiti, J. P. Fernandez-Palacios, G. Otero, C. Neumeyr, G. Delrosso, S. Bhat, K. Solis-Trapala, and G. Parladori, "Multi-Tb/s sustainable MAN scenario enabled by VCSEL-based innovative technological solutions," Proc. SPIE 11308, 113080G (2020).

17. M. Rapisarda, A. Gatto, P. Martelli, P. Parolari, C. Neumeyr, M. S. Moreolo, J. M. Fabrega, L. Nadal, and P. Boffi, "Impact of chirp in high-capacity optical metro networks employing directly-modulated VCSELs," Photonics 5, 51 (2018).

18. G. Delrosso and S. Bhat, "Development and scalability of a $2 \mathrm{~Tb} / \mathrm{s}$ data transmission module based on a $3 \mu \mathrm{m}$ SOl silicon photonics platform," in International Conference on Transparent Optical Networks (2020), paper Tu.D3.7.

19. M. S. Moreolo, J. M. Fabrega, and L. Nadal, "Sliceable BVT evolution towards programmable multi-Tb/s networking," Electronics 8, 1476 (2019).

20. P. Parolari, A. Gatto, M. Rapisarda, C. Neumeyr, M. S. Moreolo, J. M. Fabrega, L. Nadal, and P. Boffi, "Effect of filtering in dense WDM metro networks adopting VCSEL-based multi-Tb/s transmitters," in International Conference on Transparent Optical Networks (2019), paper Th.B1.3.

21. R. Muñoz, L. Nadal, R. Casellas, M. S. Moreolo, R. Vilalta, J. M. Fabrega, R. Martínez, A. Mayoral, and F. J. Vilchez, "The ADRENALINE testbed: an SDN/NFV packet/optical transport network and edge/core cloud platform for end-to-end 5G and IOT services," in European Conference on Networks and Communications (2017).

22. "Spectral grids for WDM applications: DWDM frequency grid," ITUT Recommendation G694.1, 2012.

23. J. M. Fabrega, L. Nadal, and M. S. Moreolo, "Cost-effective coherent systems for metropolitan networks," in Optical Network Design and Modelling (ONDM) (2020).

24. A. Gatto, M. Rapisarda, P. Parolari, M. S. Moreolo, C. Neumeyr, and P. Boffi, "Long-wavelength VCSEL-based system exploiting direct DMT modulation and coherent detection for multi-Tb/s metro link," in Optical Network Design and Modelling (ONDM) (2020).

25. S. Spiga, W. Soenen, A. Andrejew, D. M. Schoke, X. Yin, J. Bauwelinck, G. Boehm, and M.-C. Amann, "Single-mode highspeed $1.5 \mu \mathrm{m}$ VCSELs," J. Lightwave Technol. 35, 727-733 (2017). 
26. C. Pulikkaseril, L. A. Stewart, M. A. F. Roelens, G. W. Baxter, S. Poole, and S. Frisken, "Spectral modeling of channel band shapes in wavelength selective switches," Opt. Express 19, 8458-8470 (2011).

27. P. S. Chow, J. M. Cioffi, and J. A. C. Bingham, "A practical discrete multitone transceiver loading algorithm for data transmission over spectrally shaped channels," IEEE Trans. Commun. 43, 773-775 (1995).

28. J. G. Proakis and M. Salehi, Digital Communications, 5th ed. (McGraw-Hill, 2008).

29. L. Martin, J. M. Fabrega, M. S. Moreolo, L. Nadal, and F. J. Vilchez, "Experimental assessment of filter narrowing effect for low bandwidth connections in EON," IEEE Photon. Technol. Lett. 29, 2027-2030 (2017).
30. L. Nadal, J. M. Fabrega, M. S. Moreolo, F. J. Vílchez, R. Casellas, R. Munoz, R. Vilalta, and R. Martínez, "SDN-enabled S-BVT for SDM optical metro/regional networks," in Optical Network Design and Modelling (ONDM) (2020).

31. R. Martínez, R. Casellas, M. S. Moreolo, J. M. Fabrega, R. Vilalta, R. Muñoz, L. Nadal, V. López, J. P. Fernández-Palacios, D. Larrabeiti, and G. Otero, "Experimental evaluation of an on-line RSA algorithm for SDN-controlled optical metro network with VCSEL-based S-BVTs," in Optical Network Design and Modelling (ONDM) (2020).

32. R. Martínez, R. Casellas, M. S. Moreolo, J. M. Fabrega, R. Vilalta, R. Muñoz, L. Nadal, and J. P. Fernández-Palacios, "Proof-of-concept validation of SDN-controlled VCSEL-based S-BVTs in flexi-grid optical networks," in Optical Fiber Communication Conference, San Diego, California, March 2019, paper W1G.5. 